\title{
The role of stress anisotropy in Quantum Wire and Quantum Dot formation
}

\author{
J.P. Silveira, M.U. González, J.M. García, L. González, Y. González, F. Briones. \\ Instituto de Microelectrónica de Madrid (CNM-CSIC). C/Isaac Newton, 8. 28760-Tres Cantos. \\ Madrid. Spain. \\ e-mail: silveira@imm.cnm.csic.es
}

Stress relaxation during growth of lattice-mismatched III-V compounds can induce the formation of nanostructures. For interfaces where group- $V$ changes, the formation of either quantum wires or quantum dots has been associated with group-V element exchange process [1]. We demonstrate the importance of the stress anisotropy of the surface structure for the formation of quantum dots or wires.

We have studied the nanostructure formation in two systems with a change in the group-V element, InAs/InP and $\mathrm{GaSb} / \mathrm{GaAs}$. We have monitored both stress evolution and surface reconstruction in [110] and [1-10] directions during growth of both systems. A higher stress in [110] direction is observed which promotes an anisotropic relaxation resulting in $\operatorname{InAs} / \mathrm{InP}(001)$ quantum wire formation while maintaining a (2x4) RHEED pattern (figure 1 ). This strong stress anisotropy is related with the As-In bond distortion in [110] direction compared with the As-As dimer along [1-10] direction [2]. This particular surface configuration, arsenic stabilized, is the standard condition for MBE growth. The growth in other surface conditions as $(4 \times 2)$ surface [1] or according to our results [3] on short range disordered surfaces can avoid this surface stress anisotropy, favouring the formation of quantum dot structures.

However during growth of the $\mathrm{GaSb} / \mathrm{GaAs}(001)$ system unexpected quantum dots are obtained. We have measured the stress evolution in [110] and [1-10] directions during the Sb-As exchange process and the ulterior quantum dot formation. The initial GaAs $(2 \times 4)$ surface evolutes under $\mathrm{Sb}$ flux to a GaSb (2x8) surface, which involves two layers of $\mathrm{Sb}$ [4]. The formation of this surface structure produces a similar increase in the stress in both directions (figure 2). When GaSb is supplied, surface reconstruction remains $(2 \times 8)$ and the accumulated stress increases isotropically. In this case, relaxation appears simultaneously in both directions leading to the quantum dot formation (figure 3). Our results show that while the $(2 \times 4)$ InAs/InP surface reconstruction produces a stress anisotropy causing the formation of wires, the $(2 \times 8) \mathrm{GaSb} / \mathrm{GaAs}$ surface reconstruction minimizes the stress anisotropy leading to the formation of dots under relaxation.

This work was partially supported by Spanish CICYT under project TIC99-1035-C02. This work was partially supported by the Nanomat project of the EC Growth Programme, contract $n^{\circ}$ G5RD-CT-2001-00545.

[1] H. Yang, P. Ballet, G.J. Salamo. J.Appl.Phys. 89, 7891 (2001).

[2] J.M. García, L. González, M.U. González, J.P. Silveira, Y. González, F. Briones. J. Cristal Growth. 227-228, 975 (2001).

[3] L. González, J.M. García, R. García, F. Briones, J. Martínez-Pastor, C. Ballesteros. Appl. Phys. Lett. 76, 1104 (2000).

[4] L. J. Whitman, B.R. Bennet, E.M. Kneedler, B.T. Jonker, and B.V. Shanabrook, Suf. Sci. 436, L707 (1999). 


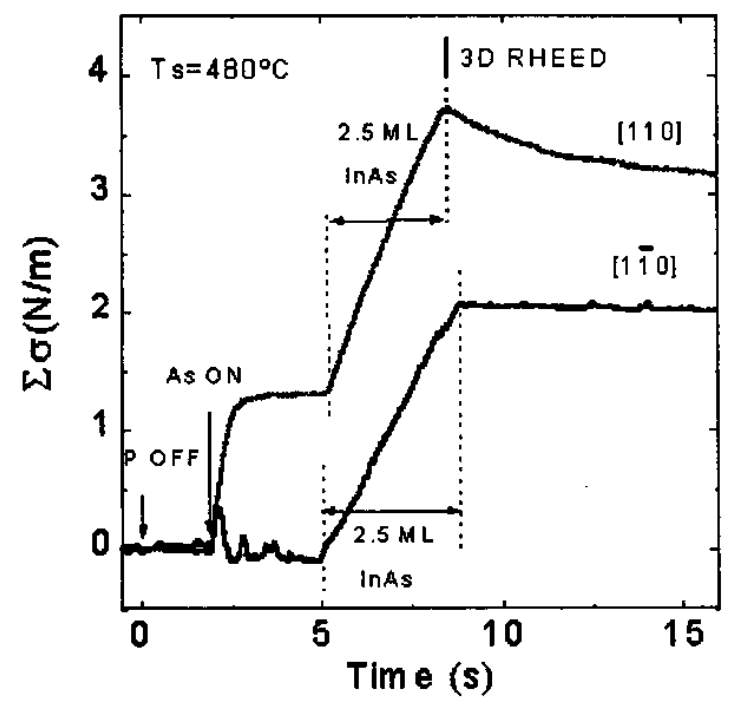

Figure 1. In situ stress measurement during InAs/InP(001) growth. The stress anisotropy between both directions is clearly observed. After $2.5 \mathrm{ML}$, relaxation takes place only in [110] direction due to quantum wire formation.

Figure 2. Accumulated stress evolution of $\mathrm{GaAs}(001)$ surface at $570^{\circ} \mathrm{C}$ under $\mathrm{Sb}$ flux (BEP $\left.=1 \times 10^{-6} \mathrm{mbar}\right)$ and recovery under As flux $\left(B E P=4 \times 10^{-6}\right.$ mbar) for [110] and [1-10] directions.
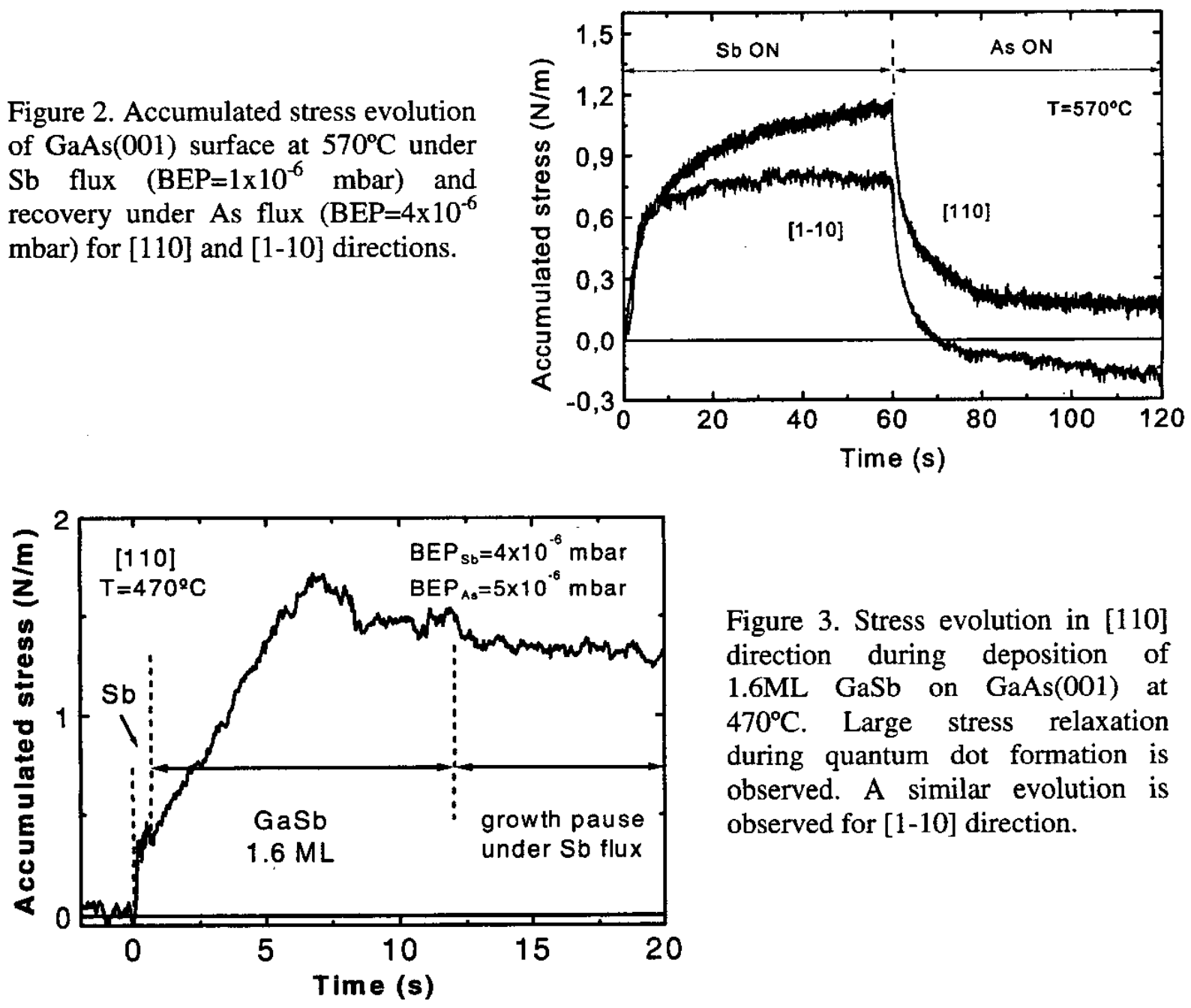

Figure 3. Stress evolution in [110] direction during deposition of $1.6 \mathrm{ML} \mathrm{GaSb}$ on $\mathrm{GaAs}(001)$ at $470^{\circ} \mathrm{C}$. Large stress relaxation during quantum dot formation is observed. A similar evolution is observed for [1-10] direction. 